CRÍTICA, Revista Hispanoamericana de Filosofta

Vol. XXIV, No. 72 (diciembre 1992): 73-101

\title{
LA DEFINICIÓN DE NÚMERO EN GOTTLOB FREGE*
}

\author{
Alfonso Ávila del Palacio \\ Universidad Juárez \\ del Estado de Durango
}

\section{Introducción ${ }^{1}$}

Hoy día se conocen muchas cosas acerca de los números: los matemáticos han ido descubriendo desde la antigüedad sus múltiples propiedades y relaciones; y hoy se aceptan varias clases de números como los primos, los irracionales, los imaginarios, los transfinitos, etc.; y se sabe cómo usarlos para muy variados propósitos prácticos, como mandar hombres a la luna o construir puentes. Sin embargo, hasta donde conozco, nunca

* El presente artículo es un extracto ligeramente revisado y actualizado del primer capítulo de mi disertación doctoral. Para la elaboración particular de estas ideas recibr la estimulante y minuciosa asesoría de Raúl Orayen, así como los atinados comentarios de Alejandro Garciadiego, León Olivé, Carlos Álvarez, Adolfo García de la Sienra, Mark Platts y José Antonio Robles, sin los cuales este trabajo no hubiera llegado a la mínima claridad que pueda poseer.

${ }^{1}$ En este trabajo, como es habitual, uso el doble entrecomillado para encerrar títulos de artículos, citas textuales no sangradas y para indicar un sentido peculiar del término entrecomillado, así como también, al igual que Frege, para expresar y resaltar el referente único de una expresión compleja de naturaleza funcional. Uso el entrecomillado sencillo, como Frege, para referirme a la expresión misma que queda entrecomillada. Uso las letras cursivas para escribir títulos de libros, palabras en algún idioma diferente al español y, como es habitual en varios de los autores trabajados, para resaltar el término escrito de ese modo. He reservado los corchetes para intercalar mis propias palabras en el interior de alguna cita textual de otro autor. 
antes de los Fundamentos de la aritmética de Gottlob Frege se había detenido alguien de una forma tan seria y sistemática a tratar de contestar la pregunta: ¿qué son al fin de cuentas los números? Es por eso, justamente, que nuestro estudio se enfoca a tratar de elucidar la respuesta dada por Frege en la obra mencionada.

Habrá que aclarar que no me detendré a examinar con el mismo cuidado todo lo que Frege dice sobre los números en esa u otras obras; particularmente, no diré nada nuevo sobre su forma particular de reconstruir la aritmética; en cambio, dedicaré un buen espacio a discutir las distinciones conceptuales que le sirven de base para su definición de número. De hecho, Frege mismo no obtiene su noción de número a partir de su reconstrucción de la aritmética; sino, más bien, "intentamos ver [dice] si las propiedades conocidas de los números se derivan de nuestra definición de número" (Frege, 1884, inc. 70). Para él, deducir lógicamente las leyes de la aritmética a partir de su definición, si ésta no puede omitirse, es una prueba de que dicha definición da en el blanco; pero, previamente a ese trabajo que intentó en sus Leyes básicas de la aritmética, discutió con bastante amplitud el aparato conceptual que utilizó en toda su obra. Así pues, trataré de mostrar que un análisis que aclare sus distinciones conceptuales nos ayudará a entender lo que encierra su definición de número de manera más apropiada que hasta ahora.

\section{Definición fregeana de número}

En Los fundamentos de la aritmética Frege comienza por revisar algunas ideas sobre el número que se encontraban dispersas en las obras de autores como Euclides, Mill, Berkeley, Leibniz, Kant, Jevons, etc., a la luz de lo que le parece incuestionable con respecto a la naturaleza de los números. En primer lugar considera que, en general, los conceptos y objetos matemáticos poseen una fuerte determinación y solidez que contrasta con 
las imágenes mentales que surgen confusamente de impresiones internas y sensoriales. Esto descalifica, para Frege, cualquier intento subjetivista de apoyar la noción de número en las percepciones sensoriales como lo hace Mill, o incluso en las intuiciones kantianas.

En segundo lugar, considera que hay una confusión en las ideas de Euclides, apoyadas en las de Aristóteles, cuando aquél afirma que "un número es una multitud compuesta de unidades [. . . donde] la unidad es aquello en virtud de lo cual cada cosa que existe se llama uno" (Euclides, defs. lib. VII); o, en palabras de Aristóteles, "la unidad es el principio del número en tanto que número, [y] el número es una multitud de mónadas" (Aristóteles, lib. X, cap. 1). En efecto mediante las unidades se individualiza cada cosa que existe $y$, en ese caso, afirma Frege, el número 5 , por ejemplo, sería la suma de cinco unidades o mónadas diferentes; de tal suerte que tendríamos que expresarlo de la siguiente forma: $5=1 a+1 b+1 c+1 d+$ le, donde $1 a \neq 1 b \neq 1 c$, etc. Sin embargo, matemáticamente $5=1+1+1+1+1$, donde $1=1$. Por lo tanto, concluye Frege, hay que distinguir el número 1 , de las unidades; el número 1 es único, mientras que las unidades pueden ser muchas. En ese sentido, el 1 es un objeto; mientras que unidad es una propiedad de los objetos tomados individualmente.

En tercer lugar, en contra de la teoría empirista de J.S. Mill, Frege observa que:

El número no se abstrae de las cosas a la manera del color, el peso, la dureza; no es, en el sentido de éstos, una propiedad de las cosas $[\ldots]$ ya que si examino uno y el mismo fenómeno externo, puedo decir con igual verdad: aquí están 5 compañías y aquí están 500 hombres; con ello no se altera ni el individuo ni el agregado, sino mi denominación [como, por cierto, ya lo había observado Berkeley]; y esto sugiere, que el contenido de un enunciado de números es una afirmación, no sobre un objeto, sino sobre un concepto. (Frege, 1884, inc. 46) 
Así pues, de acuerdo con lo anterior, Frege propone la siguiente definición de número: ${ }^{2}$

por consiguiente defino:

el número que corresponde al concepto $F$, es la extensión [alcance, tamaño o amplitud] del concepto "equinumérico [o de igual cifra] en comparación con el concepto $F$ "; (Frege, 1884, inc. 68)

que no resulta del todo clara debido a que los términos empleados tienen un significado especial dado a través de la obra fregeana.

Los intérpretes relevantes de Frege como son Dummett (1963 y 1981), Sluga (1980), Resnik (1980) y Wrigth (1983), entre otros, han estado discutiendo hasta la fecha lo que Frege quiso expresar con tales términos. En particular, la expresión que ha recibido interpretaciones más diversas es, tal vez, la de "extensión de un concepto" que Frege usó muchas veces (véase Burge, 1984), pero que evadió aclarar explícitamente. Por un lado, define el número como la extensión de un concepto. Pero, por otro, en la nota correspondiente a dicha definición, presupone que se sabe lo que es la extensión de un concepto, y afirma que incluso podría evitarse el término 'extensión'; lo cual es reafirmado al final de sus Fundamentos diciendo que "en todo esto suponemos conocido el sentido de la expresión 'extensión de un concepto', [pero, insiste] no doy una importancia decisiva a la utilización de la extensión de un concepto" (Frege, 1884, inc. 107). Estos comentarios han llevado a algunos intérpretes, como Imbert, a sostener que, "si en rigor se podrían dejar de mencionar explícitamente las extensiones, puesto que están estrechamente ligadas a los conceptos, [...] se infiere que el concepto es a la vez pensado y definido extensionalmente por Frege" (Imbert, 1972, p. 179); que contrasta

2 En alemán: "ich definire demnach: die Anzahl, Welche dem Begriffe $F$ zukommt, ist der Umfang des Begriffes, 'gleichzahlig dem Begriff $F$ '". 
con los comentarios de Russell de que Frege tiene una "teoría intensional de clases [o extensiones], y considera el número como una propiedad del concepto-clase, no de la clase en extensión" (Russell, 1903, inc. 494).

Lo cierto es que Frege definió el número como la extensión de un concepto; $y$, aunque él mismo sugirió que podría evitarse lo de extensión, realmente nunca desarrolló esa posibilidad en ninguna obra posterior. Ciertamente podemos aceptar que las mismas cosas puedan decirse de forma diferente; pero eso nos obligaría, casi siempre, sobre todo en sistemas tan interrelacionados como el de Frege, a redefinir los términos empleados. Así pues, tomando las cosas tal como fueron dichas por Frege, considero que es apremiante una aclaración de la definición fregeana en términos de la extensión de un concepto, misma que desarrollo a continuación.

\section{Aclaración del vocabulario fregeano}

Con la intención de aclarar lo que Frege entiende por 'extensión de un concepto', en lo que sigue describiré una interpretación particular de los términos fregeanos 'concepto' y 'extensión', así como de otros dos estrechamente vinculados: 'función' y 'curso de valores'; interpretación que presenté y defendí en forma más amplia en Ávila (1988).

\subsection{Los términos 'Funktion' y 'Begriff'}

Para Frege una función es el referente de una expresión con espacios vacíos que es él mismo una entidad con huecos, es decir incompleta. Los objetos, en cambio, son los referentes de expresiones saturadas que son ellos mismos entidades completas. Por ello, en el sistema fregeano, las categorías de "función" y "objeto" son mutuamente excluyentes. Para que pueda completarse una entidad que tenga huecos se requiere llenar éstos con entidades completas, es decir con objetos que en ese contexto 
se llamarían argumentos; y, al hacer esto, se obtienen otros objetos que en ese contexto se llamarían valores. De esa forma, a partir de una función, podemos distinguir una vinculación en la que ciertos argumentos se asocian con determinados valores. Frege también habla de funciones de segundo nivel, es decir, funciones que toman como argumentos otras funciones. Sin embargo, aun cuando Frege mismo no lo dijo explícitamente, considero que, estrictamente hablando, una entidad incompleta no puede completarse con entidades también incompletas porque subsistirían los huecos de estas últimas; de tal manera que, al fin de cuentas, no obtendríamos un valor que es un todo cerrado.

Ahora bien, puede mostrarse sin dificultad que una misma vinculación podría lograrse de diferentes modos: por ejemplo, la vinculación del 1 con el 2 , del 2 con el 4 , del 3 con el 6 , etc. se puede obtener a partir de diferentes funciones tales como " $2 x$ ", " $4 x / 2$ ", “ $2 x^{2} / x$ ", etc.; es decir, que para vincular estas parejas de números, puedo obtener el segundo número multiplicando el primero por 2 , o multiplicándolo por 4 y dividiendo el resultado entre 2 , etc. De acuerdo con esto, una función se puede expresar mediante un esqueleto que permita vincular nombres de objetos; ya que una función es sólo una forma o un modo como pueden vincularse los objetos aludidos. Esta forma de ver las funciones fregeanas como modos de vincular objetos es, hasta donde he podido investigar, una interpretación novedosa; y, debido a esto, me permitiré ilustrar los diferentes tipos de funciones mediante una serie de diagramas que pretenden aclarar esta interpretación.

Frege clasifica las funciones, en primer lugar, de acuerdo con el número de objetos o argumentos que requieren para producir su valor correspondiente: hay funciones de uno, dos o más argumentos. En segundo lugar, clasifica las funciones teniendo en cuenta si sus valores son objetos cualesquiera o si únicamente tienen valores de verdad: a las primeras las llama funciones no proposicionales y a las segundas, funciones 
proposicionales. De acuerdo con esto, una función no proposicional de un argumento podría estar representada de la manera siguiente:

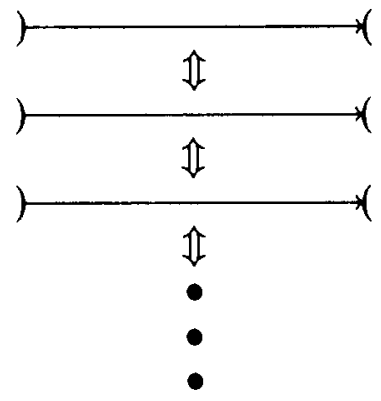

donde los espacios vacíos de la izquierda representan huecos en la función correspondiente que pueden ser llenados por diversos objetos; los espacios vacíos de la derecha representan los huecos de la función que son llenados por objetos llamados valores; las flechas horizontales representan la vinculación de cada argumento con el valor correspondiente de la función; las flechas verticales intentan expresar que se trata de una misma función que permite vincular diversos argumentos con diversos valores; y los puntos suspensivos verticales indican que esa función permite vincular no sólo tres argumentos con tres valores (de acuerdo con las tres flechas horizontales), sino, teóricamente, infinitos argumentos con infinitos valores.

- Ahora bien, ¿qué entiende Frege por 'Begriff' o 'concepto'? En la terminología de funciones, los conceptos son funciones proposicionales de un solo argumento que generan el valor "verdad" cuando toman como argumentos los objetos que caen bajo el concepto, y producen el valor "falso" cuando toman como argumentos objetos que no caen bajo el concepto. Así, por ejemplo, " $x$ es hombre" es una función-concepto en tanto que es una manera de vincular "Juan" con la verdad, "Felipe" con la verdad, "la luna" con lo falso, etc.; en tanto que la verdad es lo que se obtiene al completar la función " $x$ es hombre" con los 
argumentos "Juan" y "Felipe", y lo falso es lo que se obtiene al completarla con argumentos como "la luna". En esos términos, un concepto o función proposicional de un argumento se podría representar de la siguiente manera:

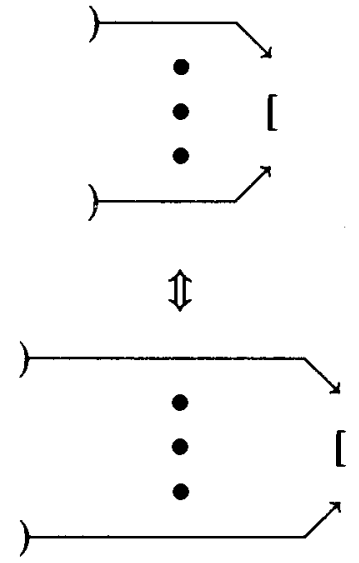

donde las líneas más cortas generan la verdad para los argumentos correspondientes y las líneas más largas generan lo falso, tratando de expresar que los argumentos que generan la verdad tienen una vinculación más estrecha con la función, puesto que son los objetos que caen bajo la función-concepto.

Para no abusar de los diagramas, creo que con los anteriores bastará para aclarar de qué forma se pueden expresar con ellos las diferentes clases de funciones. Sólo quisiera añadir que para representar diferentes funciones que permitan vincular los mismos objetos se podría usar diagramas que tuvieran la misma figura, pero mientras uno estuviera formado por líneas sencillas, otro estaría formado por líneas onduladas, otro por líneas punteadas, etc., tratando de expresar que las diferentes formas de vincular los mismos objetos, por tener, según el mismo Frege, un carácter intensional, pueden tener diferencias muy sutiles. Aunque, habría que recalcar que, estrictamente hablando. en el sistema fregeano no podemos hablar de los conceptos; 
ya que al ser entidades incompletas no pueden aparecer como argumentos en ninguna función con la cual se quiera predicar algo de ellos.

Por una cierta necesidad lingüística [afirmó Frege], mi expresión, tomada literalmente, no corresponde a veces al pensamiento, al nombrar un objeto cuando se quiere significar un concepto. Me hago plenamente consciente de apelar en estos casos a la comprensión bienintencionada del lector. (Frege, 1892, p. 54)

De hecho, esto es sobre todo porque el objeto, en tanto que es completo, no es capaz de representar cabalmente algo incompleto; es decir, no puede ser una imagen idónea de algo que es contrario a ella misma. Un representante idóneo, como afirma Wittgenstein en el Tractatus, debe tener algo en común con su representado. Pero, si al predicar de un concepto no predicamos de éste ientonces de qué estaríamos predicando? Así pues, todo esto sugiere que Frege ha quedado atrapado en sus propias redes, y que, como dice Valdivia (1984), dentro del sistema fregeano no puede decirse nada sobre los conceptos.

Terence Parsons (1984) ha propuesto otra solución que consiste en divorciar la sintaxis superficial del lenguaje natural, de su sintaxis lógica preservando, por supuesto, una mínima relación entre ellas. Para ello sostiene que Frege, "cuando explica su punto de vista, prácticamente siempre habla informal o metafóricamente o de una manera indirecta" (Parsons, 1984, inc. IV). Me parece que ésta es una vía interesante, y Frege mismo da pie para ella cuando apela a la "comprensión bienintencionada del lector" (Frege, 1892, p. 54); pero creo que este terreno es muy resbaladizo ya que no siempre es claro en qué momentos Frege está hablando metafóricamente, y podríamos fácilmente caer en la tentación de acomodar las afirmaciones fregeanas a nuestra conveniencia. Por ello, en vez de profundizar en esta posible solución, propondré en seguida una solución más sencilla y, según creo, más cercana a la propuesta 
del mismo Frege ya que nos permite hablar, dentro de un sistema fregeano ligeramente ampliado, al menos, de la parte de los conceptos que se necesita para la definición fregeana del número.

Así pues, si por un lado los conceptos y los objetos son categorías mutuamente excluyentes, y, por otro, Frege se permite hablar de los conceptos mediante objetos representativos y debe apelar a la comprensión bienintencionada del lector, pienso que el sistema fregeano podría corregirse sin forzarlo excesivamente proponiendo la existencia de ciertas "entidades conceptuales" que no serían ni conceptos ni objetos, sino algo lógicamente más simple; es decir, aquello que comparten un concepto y el objeto, llamémosle conceptual, que representa dicho concepto cuando predicamos de él. "Caballo", por ejemplo, sería la entidad conceptual que comparten el concepto "_ es caballo" con el objeto conceptual "el concepto caballo". Estas entidades conceptuales, al parecer, no se dan en forma aislada, sino siempre como parte de un concepto o de un objeto conceptual.

De acuerdo con lo anterior, cuando predicamos de un concepto tendríamos que aclarar que en realidad predicamos de la "entidad conceptual" que comparte con el objeto que lo representa; y, al parecer, eso es suficiente. El concepto, en tanto que es predicativo, es decir incompleto, se nos escapa; pero, eso no interesa con respecto a la definición fregeana del número, como trataremos de mostrarlo en la sección 4. Por supuesto, lo mismo valdría, mutatis mutandis, para las funciones en general: el aspecto de ellas sobre el que no podríamos hablar es su naturaleza no saturada. De esta forma y de acuerdo con los diagramas que propuse para expresar gráficamente los conceptos y las funciones, un objeto conceptual (o funcional) que represente un concepto (o función) podríamos expresarlo mediante un cuadrado que encierre el diagrama del concepto (o de la función) correspondiente (como, de hecho, lo haremos en el diagrama de la definición fregeana del número 2). Así quedaría expresado 
que dicho objeto conceptual (o funcional) es un todo cerrado que, sin embargo, comparte algo con el concepto (o la función) que representa. En esos términos, el referente de la figura que comparten los diagramas del concepto (o de la función) y del objeto que los representa sería la "entidad conceptual" (o funcional) correspondiente.

La existencia de estas nuevas entidades parece sugerirla la circunstancia de que en el lenguaje ordinario la expresión de un concepto como, por ejemplo, '_ es un caballo' comparte algo con la expresión 'el concepto caballo' con la cual queremos hablar de dicho concepto; a saber, la expresión caballo. Recordemos que Frege defiende frente a Kerry (Frege, 1892) que pueden sugerirse distinciones ontológicas a partir de distinciones lingüísticas. Y, por otra parte, de hecho, Frege mismo deja abierta una posibilidad así al afirmar que,

lo lógicamente simple, al igual que la mayoría de los elementos químicos, no está dado de antemano, sino que sólo se alcanza después de una labor científica. Si se descubre algo que es simple, o que al menos hasta el momento debe ser considerado como simple, habrá que acuñar para ello una denominación. (Frege, 1892, p. 43)

Así pues, considero que proponer estas entidades no violenta el sistema fregeano y, en cambio, lo hacen más inteligible.

\subsection{Los términos 'Werthverlauf' y 'Umfang'}

Ahora bien, entendiendo así el término 'concepto', ¿qué debe entenderse por 'Umfang' o 'extensión de un concepto'? Frege sostiene que "podemos designar como extensión de un concepto el curso de valores de una función de un argumento cuyo valor para cada argumento es un valor de verdad" (Frege, 1891, p. 31). En consecuencia, veamos primero lo que Frege entiende por la expresión 'Werthverlauf' o 'curso de valores'.

Frege no define lo que es un curso de valores, pero da sus condiciones de identidad en su famosa ley $\mathrm{V}$ de Las leyes bási- 
cas. En su simbología, ligeramente modernizada, la ley es como sigue: "ト $[e ́ f(e)=\dot{a} g(a)]=(x)[f(x)=g(x)]$ " (Frege, 1893, inc. 20), donde las letras acentuadas que anteceden a la expresión funcional correspondiente convierten la expresión de la función en una expresión del curso de valores de dicha función. Así pues, la ley afirma que decir que dos cursos de valores son iguales es lo mismo que decir que las funciones correspondientes arrojan los mismos valores para los mismos argumentos.

De acuerdo con esto, y teniendo en cuenta la interpretación que hemos dado para las funciones, el curso de valores sería la vinculación misma entre objetos. Las funciones son diferentes modos de vincular objetos, mientras que los cursos de valores serían las vinculaciones mismas tomadas como objetos fregeanos, es decir como referentes de expresiones saturadas. En palabras de Sluga:

la función en sí misma no es una entidad; su naturaleza es correlacionar argumentos con valores. Pero mediante la función se establece una correlación, y esa correlación puede considerarse una entidad con sus propios criterios de identidad. Esa entidad se llama curso de valores. (Sluga, 1980, p. 145)

Ahora bien, así como representamos las funciones mediante diagramas, los cursos de valores podrían expresarse de la siguiente manera:

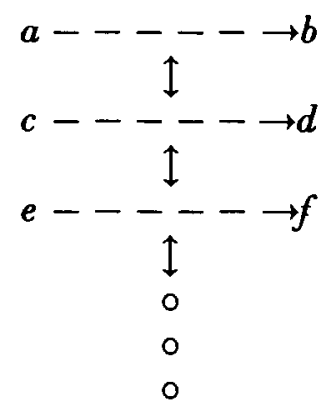


donde las letras representan objetos; las flechas entrecortadas representan la conexión entre parejas de objetos si se trata de una función de un argumento; las flechas dobles verticales representan la unidad entre todas las parejas de objetos que pueden aparearse mediante las funciones cuyo curso de valores esté expresado en el diagrama de arriba; y los círculos sucesivos verticalmente indican que las parejas de objetos vinculados no son sólo tres, sino, teóricamente, infinitas parejas de objetos. En este diagrama he usado flechas horizontales entrecortadas y flechas dobles sencillas para expresar la vinculación misma entre objetos, independientemente de las diferentes formas mediante las cuales se puede lograr la vinculación.

Por otro lado, 'Umfang' o 'extensión', al ser para Frege el curso de valores de una función proposicional sería la vinculación entre objetos cualesquiera y los valores de verdad; es decir, entre objetos y el caer o no bajo la función proposicional. El curso de valores de una función proposicional sería la vinculación entre algunos objetos y la verdad, y otros objetos y lo falso. Lo cual significa para los conceptos, o funciones proposicionales de un argumento, que no sólo se están vinculando parejas de objetos, sino que se está agrupando a los objetos que caen por un lado y a los objetos que no caen, por otro. Así pues, podemos decir que la extensión de un concepto es un conjunto de objetos que queda definido a partir de una cierta manera de vincular únivocamente objetos singulares con valores de verdad; de tal suerte que los objetos del "conjunto" están asociados con la verdad y los que quedan fuera con lo falso. ${ }^{3}$ Para Frege sus "conjuntos" son siempre extensiones de conceptos; no están constituidos por objetos como un bosque está constituido por árboles, sino que están definidos a partir del concepto.

3 Orayen (1988) muestra que, en efecto, las extensiones de los conceptos fregeanos, en el sentido que les hemos dado arriba, cumplen los axiomas de extensionalidad y comprensión con los que comúnmente se recoge la teoría cantoriana de conjuntos. 
Ahora bien, para ilustrar en mis términos la idea de que las extensiones de los conceptos fregeanos pueden cumplir, en términos generales, las mismas funciones que los conjuntos cantorianos, me auxiliaré del diagrama que presenté para las funciones proposicionales de un argumento. Según ese diagrama, todos los objetos que caen bajo el concepto están unidos con la verdad, y de esta forma quedan agrupados. De tal suerte que el curso de valores de una función proposicional de un argumento, es un conjunto fregeano en tanto que agrupa a los objetos que caen bajo el concepto. Esto lo podríamos ilustrar con el siguiente diagrama:

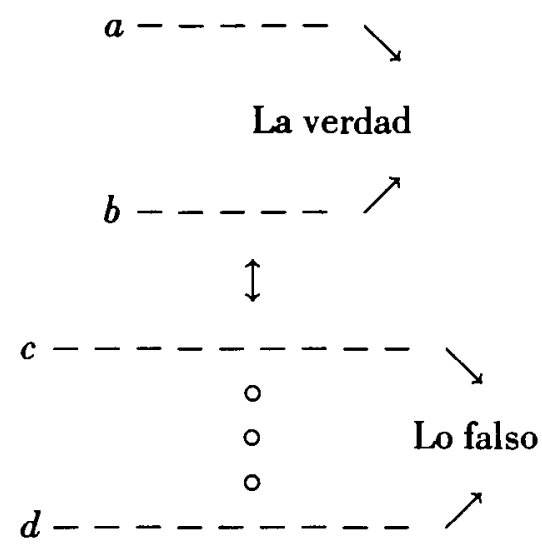

que sería análogo a la representación ordinaria de conjuntos:

$$
\ldots c \quad \begin{aligned}
& a \\
& b
\end{aligned}
$$

donde se encierran los objetos del conjunto, separándolos así de todos los demás.

En conclusión, la extensión de un concepto es una vinculación. Dado que se vinculan ciertos objetos con la verdad y otros 
con lo falso, se separan los objetos que caen bajo el concepto de los que no caen. Considero que esta interpretación es la más cercana a las ideas del propio Frege en tanto que permite conciliar varias de sus tesis al respecto: por ejemplo, permite entender: a) que las diferentes funciones que comparten un mismo curso de valores sean diferentes formas de obtener una misma vinculación entre argumentos y valores; y b) que los "conjuntos" asociados a las funciones proposicionales de un argumento, es decir las extensiones de los conceptos, se identifiquen con sus cursos de valores.

\section{4. ¿Qué es un número para Frege?}

Ahora bien, una vez aclarados en el sentido precedente los términos más problemáticos que usa Frege en su definición de número, ¿qué afirma según esto dicha definición? En primer lugar, afirma que el número es siempre el número de un concepto; lo cual significa que corresponde a las funciones proposicionales monádicas o al significado de un predicado gramatical, bajo el cual pueden caer algunas entidades. El número es, según eso, algo que tiene que ver con aquello que permite agrupar entidades, es decir, con un modo de agrupar. Sin embargo, para Frege, el número es un objeto, no una propiedad, según vimos en la sección 2 ; de tal suerte que no puede ser algo que se predique de los conceptos, sino que sólo puede ser parte de la predicación que se hace sobre ellos. De acuerdo con lo anterior, Frege sostiene que el número es una extensión, lo cual significa que es un conjunto fregeano asociado a un concepto; o, en otras palabras, que es cierta vinculación entre entidades y la verdad o lo falso, dependiendo si las entidades caen o no dentro del concepto. El número es, según esto, cierta vinculación, una vinculación, que agrupa entidades. Por último, la definición fregeana afirma que el número del concepto $F$ es la extensión del concepto "equinumérico con respecto a $F$ "; donde Frege aclara que: 
la expresión 'el concépto $F$ es equinumérico respecto al concepto $G$ ' significa lo mismo que la expresión 'hay una relación $R$ que coordina biunívocamente a los objetos que caen dentro de el concepto $F$ con los objetos que caen bajo $G^{\prime}$. (Frege, 1884, inc. 72)

lo cual significa que el número del concepto $F$ es la vinculación que agrupa los conceptos equinuméricos $\operatorname{con} F$; ya que lo que cae bajo el concepto "equinumérico con respecto a $F$ " es $F$ mismo que es un concepto y cualquier otro concepto que tenga la propiedad de que los objetos que caigan bajo él puedan ponerse en correspondencia biunívoca con los objetos que caen bajo $F$. El número es, pues, una agrupación de conceptos, ${ }^{4}$ es decir, una agrupación de modos de agrupar: es una agrupación que agrupa conceptos de acuerdo con la coordinabilidad de sus extensiones; 0 , en mis términos, es una agrupación que agrupa modos de agrupar de acuerdo con la coordinabilidad de los objetos que pueden agruparse mediante esos modos de agrupar. En ese sentido, todo concepto está agrupado en algún número de acuerdo con la coordinabilidad de los objetos que permite agrupar. Ahora bien, expresando esto mediante un diagrama, el número que corresponde al concepto ojos de mi cara sería la figura 1 , donde se representan dos argumentos que están vinculados con la verdad, por ejemplo, "el concepto ojos de mi cara" que representa a un concepto que puede agrupar dos objetos, a saber, mi ojo derecho y mi ojo izquierdo; lo cual está representado por el primer diagrama de objeto conceptual que tiene

4 Dentro de los conceptos de primer orden caen objetos y dentro de los de segundo orden caen conceptos; aunque esto, como vimos arriba, trae complicaciones en el sistema fregeano. No obstante, la definición de número habla claramente de un concepto de segundo orden, puesto que la relación de equinumerosidad Frege la define entre conceptos en (1884, inc. $71 ; 1893$, inc. 38 ). De tal suerte que si quisiéramos salvar las dificultades aludidas, los conceptos que en la definición de número se han tomado como "objetos" o entidades que caen dentro de conceptos, deberán entenderse, más bien, como objetos conceptuales en el sentido que le dimos a esta expresión en la subsección 3.1. 


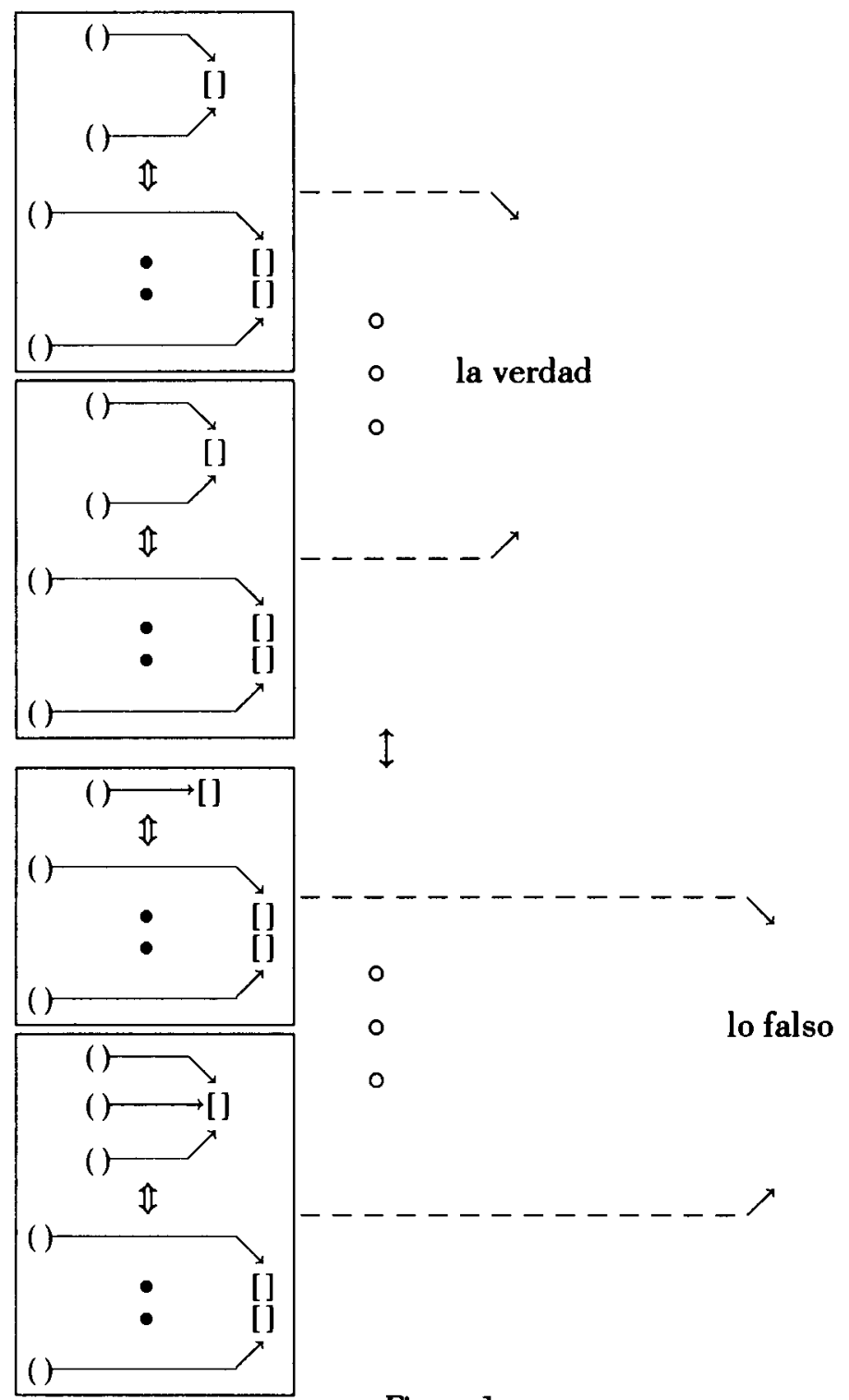

Figura 1 
dentro del cuadrado dos entradas de argumentos vinculadas con flechas cortas; y "el concepto piernas de mi cuerpo", que representa un concepto que también puede agrupar dos objetos, a saber, mi pierna derecha y mi pierna izquierda, y está representado por el segundo diagrama de objeto conceptual que también tiene dentro del cuadrado dos entradas de argumentos vinculadas con flechas cortas. Como puede mostrarse, ambos conceptos son equinuméricos ya que podemos construir una relación biunívoca que vincule, por ejemplo, mi ojo derecho con mi pierna derecha y mi ojo izquierdo con mi pierna izquierda. Ciertamente, el diagrama debería representar también todos los otros conceptos equinuméricos con esos dos; pero por razones obvias de simplicidad los he omitido. El tercer objeto conceptual está representado por el diagrama que dentro del cuadrado sólo tiene una entrada de argumento vinculada con una flecha corta y que puede ser, por ejemplo, "el concepto nariz de mi cara", ya que dentro del concepto correspondiente sólo cae un objeto, a saber, la única nariz que tengo en la cara. Este objeto conceptual está vinculado con lo falso puesto que no puede aparearse el único objeto que permite agrupar el concepto correspondiente con los dos objetos que permite agrupar el concepto ojos de mi cara. Por último, el cuadrado que encierra un diagrama que puede vincular tres nombres de objetos con la verdad representa un objeto conceptual como, por ejemplo, "el concepto rey mago"; ya que dentro del concepto correspondiente caen sólo tres objetos, a saber, Melchor, Gaspar y Baltasar. Este objeto conceptual está vinculado también con $l o$ falso; ya que los tres objetos que caen dentro del concepto correspondiente no pueden aparearse con los que caen dentro de ojos de mi cara. De la misma forma, también estarán vinculados con lo falso todos los objetos-conceptuales para los cuales no pueda construirse una relación biunívoca entre los objetos que caigan dentro de los conceptos correspondientes y los que caen dentro de el concepto ojos de mi cara. 
Si eliminamos el término 'extensión', como lo sugiere Frege en la nota a su definición, el número sería un concepto de conceptos, es decir, un modo de agrupar modos de agrupar. No obstante, como dijimos en la sección 2, Frege nunca desarrolló esa idea y lo que dijéramos al respecto sería pura especulación. Lo cierto es que, para Frege, la extensión, que es un objeto fregeano, es siempre la extensión de un concepto; y esto nos deja ver que para él ese objeto está siempre asociado a un concepto, y se da sólo a partir de éste. Frege definió el número como una extensión porque para él el número es un objeto, donde objeto y concepto son categorías excluyentes. No obstante, Frege definí́ el número como una agrupación de conceptos y, por las dificultades que vimos en la subsección 3.1, lo que puede agruparse en el sistema fregeano son más bien objetos. Ciertamente, los conceptos que agrupa un número están agrupados, no por alguno de sus rasgos intensionales o de cualquier otro tipo, sino por una de sus propiedades de carácter extensional, es decir, por una característica de sus respectivas extensiones. Todo esto ha llevado a varios de los comentadores y seguidores de la línea fregeana como Russell y Quine a definir el número, no como una extensión que agrupa conceptos, sino como una extensión que agrupa extensiones: justo las extensiones de los conceptos que agrupa la definición fregeana. De esa forma, el número se define como una extensión que agrupa extensiones $o$, si se prefiere, como un conjunto de conjuntos. Ahora bien si, para observar mejor las similitudes y diferencias en ambas definiciones, representamos también esta segunda versión con un diagrama similar al anterior, el mismo número 2 quedaría expresado como en la figura 2 , donde están representadas primero las extensiones de los conceptos "ojos de mi cara" y "piernas de mi cuerpo" vinculadas con la verdad, ya que se pueden poner en correspondencia biunívoca los objetos que caen dentro de ellos apareando, por ejemplo, el objeto $a$ con el objeto $x, y b$ con $y$; mientras que, por otro lado, no podemos aparear $[a, b]$ con $[e]$ ni con $[j, n, k]$, que son los objetos que caen dentro de los 


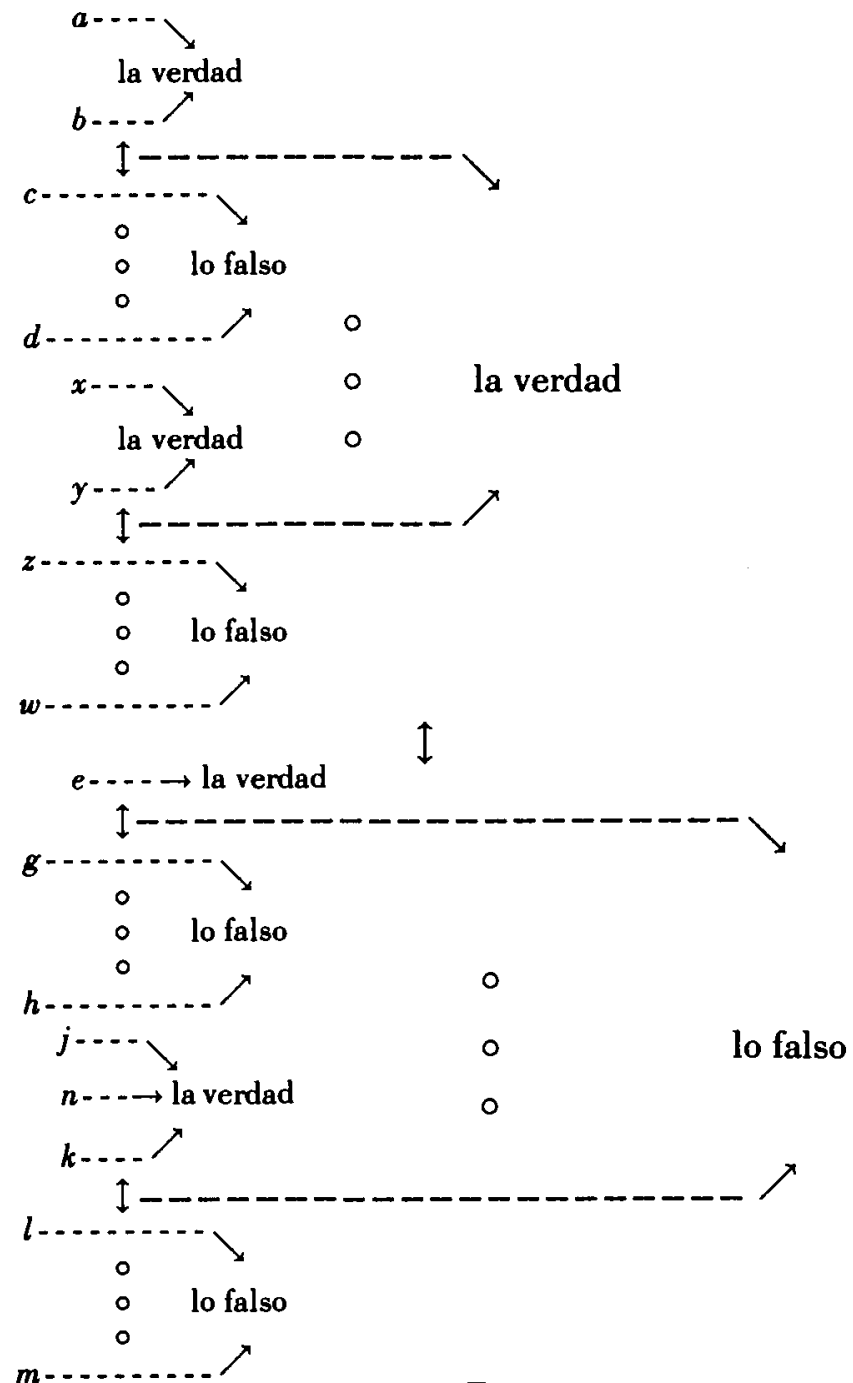

Figura 2 
conceptos "nariz de mi cara" y "rey mago", respectivamente, cuyas extensiones están expresadas y, por consiguiente, vinculadas con lo falso. Así pues, en esta versión, el número de la extensión del concepto "ojos de mi cara" sería el "conjunto" o agrupación a la que pertenece esa extensión en tanto que pueda ponerse en correspondencia biunívoca los objetos que agrupa con los que agrupan otras extensiones. En resumen, el número, según esta versión, es el número de una extensión, es decir, de una agrupación, digamos $A$; y el número de esa agrupación de objetos es la agrupación de agrupaciones que puede lograrse a partir de un concepto, digamos $G$, que podría expresarse con el predicado "ser extensión que agrupa objetos que puedan ponerse en correspondencia biunívoca con los objetos que agrupa la extensión $A$ ".

Mientras que en la versión fregeana un número es una agrupación de conceptos, en la otra versión, el número es una agrupación de extensiones. Sin embargo, en cierto sentido ambas versiones son equivalentes ya que, finalmente, en las dos un número "atrapa" directa o indirectamente los mismos conceptos y las mismas extensiones. En la versión fregeana, el número del concepto $F$ agrupa determinados conceptos dependiendo de si sus respectivas extensiones tienen o no cierta característica; y así, quedan "atrapadas" también las extensiones que tengan esa característica. Por otro lado, en la otra versión, el número de la extensión del concepto $F$ agrupa determinadas extensiones, justo aquellas que quedan "atrapadas" en la versión fregeana; pero en Frege la extensión es siempre la extensión de un concepto; así pues, de esa forma quedan también "atrapados" los conceptos asociados con esas extensiones, y éstos son justamente los que agrupa la versión fregeana. En consecuencia, en ambas versiones, el número del concepto $F$ agrupa o "atrapa" los mismos conceptos y las mismas extensiones, y por ello podrían manejarse como equivalentes haciendo los ajustes requeridos en el sistema fregeano. De hecho, la similitud estructural que puede observarse sin dificultad en los 
dos diagramas que presentamos del número 2 , así como con un tercero que representara la versión del número como un concepto de conceptos sugerida de pasada por el mismo Frege, nos sugiere que podríamos usar cualquiera de ellas haciendo los ajustes necesarios. No obstante, Frege usó claramente sólo la primera versión.

Ahora bien, entendidos así los números, Frege pretendió confirmar su idea "viendo si se pueden deducir propiedades conocidas de los números a partir de esa definición" (Frege, 1884, inc. 70). Al final de los Fundamentos y en las Leyes básicas de la aritmética, de acuerdo con sus distinciones conceptuales y las leyes de inferencia desarrolladas a partir de su Conceptografia, Frege obtiene varias de las principales proposiciones acerca de los números naturales, para lo cual incluyó como parte de la lógica su ley de la igualdad de las extensiones que hoy se sitúa dentro de la teoría matemática de conjuntos. $\mathrm{Al}$ respecto dice:

sólo puede haber discusión, por lo que alcanzo a ver, respecto de mi ley fundamental de los cursos de valores $(V)$, que quizás los lógicos todavía no consideran como cosa propia, aunque se piensa en ella cuando se habla, por ejemplo, de extensiones de concepto; (Frege, 1893, int.)

lo cual es explicable porque para Frege las extensiones están siempre formadas a partir de los conceptos; y si la lógica se encarga de los conceptos, al parecer no ve por qué las extensiones no sean también asunto de la lógica.

Su método para reconstruir la aritmética consiste en mostrar que las agrupaciones de conceptos que define como números tienen en primer lugar las mismas propiedades que los números naturales tradicionales. Para ello, definió cada número individual eligiendo un concepto al que le corresponda ese número. Por ejemplo, 0 lo define como el número que corresponde al concepto "desigual consigo mismo"; 1 , como el número que 
corresponde al concepto "igual a cero"; y 'sucesor' lo define diciendo:

Existe un concepto $F$ y un objeto $x$ que cae dentro de él, de tal tipo que el número que corresponde al concepto $F$ es $n$, y que el número que corresponde al concepto "que cae dentro de $F$, pero no es igual a $x$ " es $m$; [o, con otras palabras] que $n$ sigue inmediatamente a $m$ en la serie de los números naturales; (Frege, 1884 , inc. 76 )

y a partir de ahí deduce para los números así definidos las propiedades aritméticas más conocidas. Esto lo hace en los incisos 74 a 77 de los Fundamentos y 41 a 43 de las Leyes básicas.

En resumen, Frege proporciona una definición de los números que los vincula estrechamente con los conceptos; y además, lo definido así se comporta, al parecer, como los números naturales tradicionales. Lo primero permite ubicar los números en el panorama general de los conceptos y lo segundo refuerza la hipótesis de que los ubicados sean realmente los números que se han usado durante siglos. Por todo ello y por el entusiasta apoyo de pensadores relevantes, como Russell, la definición fregeana ha sido a menudo aceptada, aunque no por ello ha estado completamente libre de objeciones.

En primer lugar, se encuentra la gran objeción de Russell (1902) cuando le comunica a Frege que ha encontrado una contradicción en su Conceptografia en el punto en que éste considera que las funciones son los elementos indeterminados o incompletos de toda oración en la cual existen otros elementos, llamados argumentos, que pueden ser reemplazables y que son determinados y completos; siendo toda la oración (función más argumentos) algo completo o determinado (véase Frege, 1879, inc. 9). Aplicando lo anterior a los conceptos, al ser éstos casos especiales de funciones, quedarían definidos como el elemento incompleto de toda oración que al completarse con un argumento produce un valor de verdad determinado. Sin embargo, al no estipular ninguna restricción a la indeterminación 
o incompletud de las llamadas funciones, se podrían presentar algunas que generaran contradicciones como, por ejemplo el concepto propuesto por Russell, "_ es un predicado que no puede predicarse con verdad de sí mismo", sobre el cual no puede decidirse si cae o no dentro de la predicación que expresa; es decir, que al actuar como elemento reemplazable en el espacio vacio 5 el resultado no es un valor de verdad determinado. A lo cual, Frege contestó: "Su descubrimiento de la contradicción me causó una gran sorpresa. Parece entonces que una igualdad de cursos de valores no está siempre permitida, que mi ley $\mathrm{V}$ es falsa [...] y que mi combinación de signos no tiene significado en todos los casos" (Frege, 1902, p. 124). En otras palabras, eso significa que no siempre podemos pasar de un concepto a su extensión (o conjunto asociado) sin caer en contradicciones; $o$, en nuestros términos, que no todos los conceptos pueden pensarse como modos en los que se pueden agrupar objetos. Puesto que, si entendemos un 'concepto' como un modo de agrupar objetos (es decir, de formar conjuntos), es una contradicción encontrarse con un modo de agrupar que no permite agrupar (es decir, que no tiene un conjunto asociado). Viendo en estos términos las cosas, podemos apreciar la gravedad de la objeción de Russell, ya que tendríamos que redefinir lo que se entiende por 'concepto' y 'extensión', así como por los términos estrechamente vinculados de 'función' y 'curso de valores', alterando, por consiguiente, todo el sistema.

Frege propuso una solución en un apéndice al volumen II de sus Leyes básicas que consiste en restringir la ley $\mathrm{V}$ diciendo que las extensiones de dos conceptos son iguales si y sólo si para todo argumento, si éste no es igual a ninguna de las dos extensiones, los conceptos arrojarán los mismos valores. Esta solución, como lo aclara el mismo Frege, no es una definición

5 Dejando de lado la dificultad que analizamos en 3.1 de que un concepto tome el papel de argumento. 
de extensión, sino que sólo establece ciertas propiedades de las funciones de segundo nivel. Sin embargo, Lesniewski en 1938 (véase Resnik, 1980, p. 215), posteriormente Quine (1955), Geach (1956) y recientemente Resnik (1980) han mostrado que la solución de Frege no es del todo satisfactoria; y no lo es, básicamente, porque lo que necesita Frege, de acuerdo con la aclaración de la contradicción hecha arriba, es justamente redefinir sus términos. Como es bien sabido, posteriormente a Frege se han propuesto soluciones para esa dificultad del sistema fregeano que han consistido, precisamente, en diversas definiciones de conjuntos, en las cuales, como regla general, se evita implicar a los conceptos; así, por cierto, se mutila la propuesta fregeana (Russell, 1903, cap. 14 y Ávila, 1989, pp. 5662). No es nuestro propósito detenernos ahora a examinar esas soluciones, ya que lo único que intentamos hacer aquí es dar un poco de claridad a la definición fregeana de número. Con esto esperamos que, al menos, puedan verse mejor los límites y alcances de las críticas que se le han hecho.

Por otro lado, la definición de Frege ha sido atacada también en cuanto a si sus agrupaciones de conceptos son realmente los números tradicionales y no únicamente algo que se comporta como éstos. En ese sentido se encuentra, por ejemplo, el famoso artículo de Benacerraf "Lo que los números no podrían ser", que lleva a cuestionar si en efecto los números son determinados conjuntos e, incluso, si son objetos específicos, ya que pueden encontrarse otros conjuntos que los propuestos por Frege que soporten la prueba a la que sometió él mismo los suyos en las Leyes de la aritmética, es decir, básicamente, que cumplan los axiomas de Peano; lo cual sitúa a Benacerraf en la concepción estructuralista de Dedekind, y otros, que afirma que los números son sólo lugares en una serie.

A este respecto, habrá que aclarar que no deben confundirse los números aritméticos, cualquier cosa que ellos sean, y las reconstrucciones o pinturas que se han hecho de ellos, como es el caso de las propuestas por Frege, Russell, Dedekind o 
Peano mismo. En otra parte (Ávila, 1989, pp. 161-178), he hecho una sugerencia que continúo trabajando, según la cual a los números aritméticos los he llamado "números de segundo nivel" debido a que podemos verlos como pinturas de entidades extra matemáticas; mientras que a las reconstrucciones de Frege, Russell, Peano, etc., las he llamado "números de tercer nivel" porque podemos verlas, a su vez, como pinturas de los números aritméticos. Según esto, en efecto, como lo sostiene Benacerraf, Frege no puede decir que los números (de segundo nivel) son extensiones que agrupan conceptos; sino, en todo caso, que dichos números pueden verse como tal cosa. Ahora bien, si siempre podemos tener diversas pinturas de una misma cosa, los mismos números pueden verse también mediante otros esquemas, como los sugeridos por Russell, Dedekind o Peano. Creo que los esquemas (o números de tercer nivel) de Frege y Russell que presentamos arriba permiten ver lo que eso significa.

Por otra parte, al tomar un esquema particular (el de Peano, por ejemplo) como el retrato básico al que deben ajustarse los demás, ya se está tomando partido en relación con lo que son los números aritméticos. Sin embargo, creo que esta cuestión está todavía abierta de acuerdo con los trabajos de Russell (1903), Wright (1983 y 1988), Kitcher (1984) y Ávila (1989), entre otros; todos los cuales sostienen, como Frege, que hay algo más en los números aritméticos que lo puramente estructural. En resumen, considero que hay algo en Frege que no recoge el análisis de Benacerraf, $y$, por lo tanto, el ataque de éste no nulifica del todo la propuesta fregeana si ésta la vemos sólo como una forma (entre otras) de ver los números aritméticos; sin olvidar, claro está, que sus términos deben ser redefinidos, pero advirtiendo que la conexión que establece con algo extra matemático, como son los conceptos, es muy sugerente y permite atribuirles a los números aritméticos una propiedad no estructural: su conexión con conceptos específicos. 


\section{BIBLIOGRAFIA}

Aristóteles, Metaphysics, trad. W.D. Ross, en Great Books of the Western World, William Benton, Publisher, Encyclopedia Britannica, 1952 , t. VIII, pp. 499-626.

Ávila, Alfonso, 1988, "Sobre la noción fregeana 'extensión de un concepto'", Análisis Filosofico (SADAF, Argentina), vol. VIII, no. 1.

__, 1989, Las matemáticas y las ciencias empiricas: ¿qué podrían ser los números?, tesis doctoral, UNAM, México.

Benacerraf, Paul, 1965, "What Numbers Could not Be", en P. Benacerraf y H. Putnam (comps.), Philosophy of Mathematics, 2a. ed., Cambridge University Press, Cambridge, 1983, pp. 272-294.

Burge, Tyler, 1984, "Frege on Extension of Concepts from 1884 to 1903", The Philosophical Review, vol. XCIII, no. 1, enero.

Dummett, Michael, 1963, Frege Philosophy of Language, Duckworth, Londres.

- 1981, The Interpretation of Frege's Philosophy, Duckworth, Londres.

Euclides, The Thirteen Books of Euclid's Elements, trad. Thomas L. Heath, en Great Books of the Western World, William Benton, Publisher, Encyclopedia Britannica, 1952, t. II, pp. 1-396.

Frege, Gottlob, 1879, Begriffsschrift, en Jean van Heijenoort (comp.), From Frege to Gödel, Harvard University Press, Cambridge (Mass.), 1967, pp. 1-82.

_- 1884, Die Grundlagen der Arithmetik, Hildesheim, Alemania, 1961.

- 1 1891, "Function and Concept", en Peter Geach and Max Black (comps.), Translations from the Philosophical Writings of Gottlob Frege, 3a. ed., Basil Blackwell, Oxford, 1980, pp. $21-41$.

_ 1892, "On Concept and Object", en Peter Geach and Max Black (comps.), Translations from the Philosophical Writings of Gottlob Frege, 3a. ed., Basil Blackwell, Oxford, 1980, pp. 42-55. _, 1893, The Basic Laws of Arithmetic, trad., ed. e introd. Montgomery Furth, University of Califormia Press, Berkeley/Los Angeles, 1964.

— 1902, "Letter to Russell", en Jean van Heijenoort (comp.), From Frege to Gödel, Harvard University Press, Cambridge (Mass.), 1967, pp. 126-128. 
Geach, Peter, 1956, “On Frege's Way Out”, en E.D. Klemke (comp.), Essays on Frege, University of Illinois Press, Urbana/Chicago/ Londres, 1968, pp. 502-504.

Imbert, Claude, 1972, "Estudio de los Fundamentos de la aritmética de Frege", en G. Frege, Fundamentos de la aritmética, trad. Ulises Moulines, Editorial Laia, Barcelona.

Kitcher, Philip, 1984, The Nature of Mathematical Knowledge, Oxford University Press, Nueva York/Oxford.

Orayen, Raúl, 1988, "Los Werthverlauf de Frege y la teoría de conjuntos", Análisis Filosbfico (SADAF, Argentina), vol. VIII, no. 1, pp. 1-18.

Parsons, Terence, 1984, "Why Frege should not have said 'the Concept Horse is not a Concept'?", Mathematical Research, memoria del Congreso Internacional sobre Frege, Schwerin (GOR), septiembre 10-14.

Quine, W. Van Orman, 1955, “On Frege's Way Out”, en E.D. Klemke (comp.), Essays on Frege, University of Illinois Press, Urbana/Chicago/Londres, 1968, pp. 485-501.

Resnik, M.D., 1980, Frege and the Philosophy of Mathematics, Cornell University Press, Ithaca/Londres.

Russell, Bertrand, 1902, "Letter to Frege", en Jean van Heijenoort (comp.), From Frege to Gödel, Harvard University Press, Cambridge (Mass.), 1967, pp. 124-125.

- 1903, The Principles of Mathematics, George Allen and Unwin Ltd., Londres.

Sluga, Hans, 1980, Gottlob Frege, Routledge and Kegan Paul, Londres.

Valdivia, Lourdes, 1984, "Lo indecible y sus raíces categoriales en la teorfa de Frege", en S. Álvarez et al. (comps.), Lógica y filosofia del lenguaje, Ediciones Universidad de Salamanca, Salamanca, vol. II, pp. 297-315.

Wright, Crispin, 1983, Frege's Conception of Numbers as Objects, Aberdeen University Press.

1988, "What Numbers Can Believably Be: a Reply to Hartry Field", Revue Internationale de Philosophie, vol. 42, no. 167, pp. 425-473. 


\section{SUMMARY}

In this paper, Frege's definition of numbers is analyzed and a new pictorial interpretation of this is proposed based on the linguistic analysis of Frege's terms 'Funktion', 'Begriff', Werthverlauf' and 'Umfang'. Here, we argue that 'Funktion' means "way to link objects", 'Begriff' means "way to group objects", 'Werthverlauf' means "link of objects" and 'Umfang' means "group of objects". Frege's system does not permit a link (or group) of ways to link (or ways to group) to exist, because a way to link (or to group) is not an object. However, Frege's definition of numbers tells us of a group of ways to group objects. So, in this work a solution is proposed that is an ontological distinction based on a linguistic distinction. If we say that "- is a horse" is a way to group objects, and that "the concept horse" is a way to group which is in the role of an object, then one can think that the term 'horse' expresses some entity that is more basic and that it is what enables us to speak about concepts; it implies that a group of ways to group exists.

To Frege, the number is a group of ways to group which permits us group the same number of objects. For example, the number 2 would be the group of ways to group pairs of objects. But this group is formed on the basis of an extensive characteristic of the ways to group: the number of elements of the groups which those ways to group permit. Then, Russell's definition of numbers like groups of groups permits to catch the same groups and same ways to group as Frege's definition.

However, Frege's definition of numbers is not indisputable. Russell's paradox compels us to make new definitions of Frege's terms 'Funktion', 'Begriff', 'Werthverlauf' and 'Umfang', because he finds a way to group which dues not permit to form a group. Finally, the objection of Benacerraf compels us to distinguish the arithmetical numbers from the pictures of arithmetical numbers, like those made by Frege, Russell, Dedekind or Peano. 